\title{
Conversion among photo-oxidative products of polypropylene in solid, liquid and gaseous states
}

\author{
Xuan Liu and Rui Yang * (1)
}

\begin{abstract}
During aging of polymers, oxidized species on macromolecular chains in solid state, volatile degradation products in liquid state and gaseous degradation products in gaseous state are often investigated separately. The conversion among these products is not especially concerned and biased results may be obtained based on the products in a single state. In this paper, photo-oxidative products of commercial polypropylene (PP) and unstabilized PP in solid, liquid and gaseous states were investigated by using Fourier transform infrared spectroscopy (FTIR), pyrolysis-gas chromatography/mass spectrometry (Py-GC/MS) and gas chromatography (GC). By comparing the formation profiles, conversion among the photo-oxidative products in three states was traced. During photo-oxidative aging, the main chains of PP were first oxidized to form carbonyl species in solid state, or fractured to form volatile alkenes as liquid. With the proceeding of aging, the oxidized main chains fractured to form small molecules, resulting in the conversion of oxidized species from solid state to liquid and gaseous states. When the aging degree was extremely high, the accumulation of liquid oxidized products was limited due to migration and condensation. Therefore, both the carbonyl index $(\mathrm{Cl})$ and the concentrations of volatile oxidized products were increased first and then decreased, while the concentrations of gaseous products kept increasing all along.
\end{abstract}

Keywords: Polypropylene, Photo-oxidative aging, Conversion, Aging degree

\section{Introduction}

During the aging process of polymers, oxidation and degradation of macromolecular chains happened, leading to the deterioration of mechanical properties [1-3]. The aging products of polymers exist in solid, liquid and gaseous states. Solid products are resulted from the oxidation of main chains $[4,5]$. Liquid products are small molecular degradation products, which can be absorbed in the aged polymers or volatilize [6]. Gaseous products are smaller molecules, which are in gaseous state at room temperature and easily migrate out [7]. Oxidized main chains continuously fractured to generate liquid and gaseous

*Correspondence: yangr@mail.tsinghua.edu.cn

Department of Chemical Engineering, Tsinghua University,

Beijing 100084, People's Republic of China molecules. The dynamic process results in continuous change of products in three states. Therefore, studying the products in a single state is not enough to reflect the whole aging process.

Most researches focused on the changes of solid polymers before and after aging. For instance, changes of functional groups, chromophores, molecular weight, crystallinity and morphology were often used to evaluate the aging degree of polymers [8-12]. Some researchers turned their eyes to small molecular degradation products [13]. Carlsson, Wiles and Philippart contributed to the identification of volatile and gaseous products of polypropylene (PP) in various aging conditions [14-16]. Combination of selective isotopic labeling with solidphase microextraction or cryotrapping gas chromatography/mass spectroscopy made it possible to confirm the 
original position of carbons on the main chains entering the volatile products [17]. Egerton et al. [18-21] analyzed the generation of $\mathrm{CO}_{2}$ in real time during photo-oxidation of a series of polymers, such as acrylic paint, low density polyethylene (LDPE), poly(vinyl chloride) and polyethylene terephthalate.

When the stabilities of polymers were compared based on macromolecular products or small-molecular degradation products separately, there might be conflicting results. For example, rare volatile oxidized products were generated during photo-oxidation of high density polyethylene composites, but the carbonyl index (CI) of the solid sample was rather high [22]. Another example was found in LDPE nanocomposites. LDPE/ZnO nanocomposites had lower $\mathrm{CI}$ but much higher concentration of $\mathrm{CO}_{2}$ than $\mathrm{LDPE} / \mathrm{TiO}_{2}$ nanocomposites after photo-oxidative aging [23]. These facts remind us that the products in a single state can only provide information related to aging properties from a single aspect. However, there are few works on the whole aging procedure, including changes of solid, liquid and gaseous states together. In order to understand aging mechanism comprehensively, studying the conversion among aging products in three states is of great importance.

In this paper, photo-oxidative products of two PPs with different stabilities in solid, liquid and gaseous states were studied. Different induction periods indicated different generation times of these products. Conversion among the products in three states was traced. Overview of the aging products in three states was an effective way to obtain comprehensive information of the whole aging process and avoid possible biased evaluation of the aging degree and relative stability of polymers.

\section{Experimental \\ Materials}

Two kinds of PPs were used: commercial PP pellets (F401, purchased from Sinopec, with stabilizers' package containing UV stabilizers and/or antioxidants, marked as CPP) and unstabilized PP powders (supplied by PetroChina Panjin Petrochemical Company, without any additives, marked as UPP). Both pellets and powders were hot-pressed at $190{ }^{\circ} \mathrm{C}$ into films (thickness of $0.3 \mathrm{~mm}$ ).

\section{Photo-oxidative aging}

Photo-oxidative aging was conducted in a Q-Sun Xe-3 chamber (Q-Lab Corporation). A film $(40 \mathrm{~mm} \times 15 \mathrm{~mm}$ ) was put into a sealable quartz tube before aging (Fig. 1). The intensity of UV irradiation was $0.35 \mathrm{~W} /$ $\mathrm{m}^{2} / \mathrm{nm} @ 340 \mathrm{~nm}$. The temperature was $60{ }^{\circ} \mathrm{C}$ and the time intervals were $48,96,144,288,384$ and $480 \mathrm{~h}$. Before aging, the valve was closed to seal the tube. After aging, the valve was opened and the gas in the tube

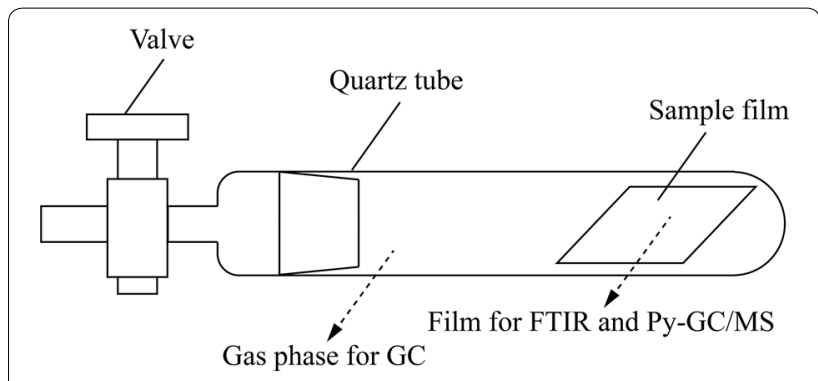

Fig. 1 Schematic diagram of reaction device

was extracted for the gas chromatography (GC) measurement. Then the film was taken out for the Fourier transform infrared spectroscopy (FTIR) and pyrolysisgas chromatography/mass spectrometry (Py-GC/MS) measurements.

\section{FTIR measurement}

FTIR measurement was conducted to detect the oxidized macromolecules in solid state. Transmission spectra were obtained by using a Thermo-Nicolet iS10 FTIR spectrometer. For attenuated total reflection (ATR) mode, an accessory with a diamond crystal was used. CI was calculated by dividing the peak area at $1714 \mathrm{~cm}^{-1}$ to the peak area at $2722 \mathrm{~cm}^{-1}$ [24]. Three measurements were carried out for each film and the results were averaged.

\section{Py-GC/MS measurement}

Py-GC/MS measurement was conducted in a gas chromatography/mass spectrometer (GC/MS-QP2010 SE, Shimadzu) installed with an EGA/PY-3030D multi-shot pyrolyzer (Frontier Laboratories) to detect the volatile degradation products absorbed by the film. $10.0 \mathrm{mg}$ film or ethanol solution of the condensed liquid droplets on the tube wall was heated at $300{ }^{\circ} \mathrm{C}$ for $30 \mathrm{~s}$ and the evaporated components went through an Ultra Alloy- 5 column. Limited by the stationary phase of the column, components with carbon number less than six might not be separated completely. The peak area of a component in the flash evaporation-gas chromatogram was calculated to represent the relative concentration of the component. Two measurements were carried out for each film and the results were averaged.

\section{GC measurement}

GC measurement was conducted in a 7890B GC system (Agilent Technologies) to detect the gaseous degradation products around the film in the tube. $200 \mu \mathrm{L}$ gas were extracted from a tube by an injector and injected into the GC system, through a G3591-81023 column. According to the separation capacity of the column, only small 
gaseous molecules like $\mathrm{H}_{2}, \mathrm{~N}_{2}, \mathrm{O}_{2}, \mathrm{CO}, \mathrm{CO}_{2}$, and alkanes and alkenes with carbon number less than three could be detected. A flame ionization detector and a thermal conductivity detector were used. The peak area of a component in the gas chromatogram was calculated to represent the relative concentration of the component.

\section{Results and discussion}

\section{Macromolecular products}

CI mainly reflected the accumulation of carbonyl products on the macromolecular chains in solid state. CI calculated from ATR spectra showed the local oxidation degree on the surface of the film, while CI calculated from transmission spectra showed the average oxidation degree in the bulk polymer.

ATR spectra, transmission spectra and corresponding CI of two PPs are shown in Figs. 2 and 3. On the surface (Figs. 2a, b and 3a), CPP had an induction period of at least $96 \mathrm{~h}$, while UPP was oxidized from the very beginning of aging. After the induction period, CPP was oxidized at a rate $\left(0.10 \mathrm{~h}^{-1}\right)$ similar to UPP $\left(0.11 \mathrm{~h}^{-1}\right)$ before $288 \mathrm{~h}$. After that, the increase of CI in UPP slowed down. Two PPs seemed to reach the similar oxidation degree after $480 \mathrm{~h}$. In the bulk (Figs. 2c, d and 3b), UPP was also oxidized from the very beginning of aging, while CPP exhibited an induction period of at least $96 \mathrm{~h}$. The oxidation rate of CPP after $288 \mathrm{~h}\left(0.04 \mathrm{~h}^{-1}\right)$ was the same to that of UPP before $288 \mathrm{~h}\left(0.04 \mathrm{~h}^{-1}\right)$. After $288 \mathrm{~h}$, the CI of UPP decreased.

The decrease of the CI in Fig. 3b seemed unreasonable, since the oxidation of PP was an accumulation process of the oxidized species. Therefore, some highly oxidized species might be lost. Considering that UPP was easier to be oxidized than CPP, it reached a high oxidation degree first. At the late stage of aging (after $288 \mathrm{~h}$ ), severely oxidized fragments might drop from the surface and cause the decrease of CI [25-27]. The change of CI of UPP in Fig. 3a supported the speculation. The surface oxidation degree of UPP after aging for $288 \mathrm{~h}$ also showed a lower increasing rate than that of CPP. In this case, although the aging degree of UPP was evidently higher than that of CPP, the CI of UPP was close to or even lower than that of CPP after $288 \mathrm{~h}$. Thus, the biased evaluation of the aging degree was obtained.

In addition, different sensitivities of ATR spectra and transmission spectra were observed. Despite the same difference between the oxidation degrees of two PPs after the same aging time, the differences between CI of two PPs were not the same from ATR spectra and transmission spectra. For instance, after aging for $288 \mathrm{~h}$, the CI from ATR spectra of UPP was about 1.4 times of that of CPP, while the CI from transmission spectra of UPP was about 4.2 times of that of CPP. Obviously, transmission spectra were more sensitive to the different oxidation degrees.

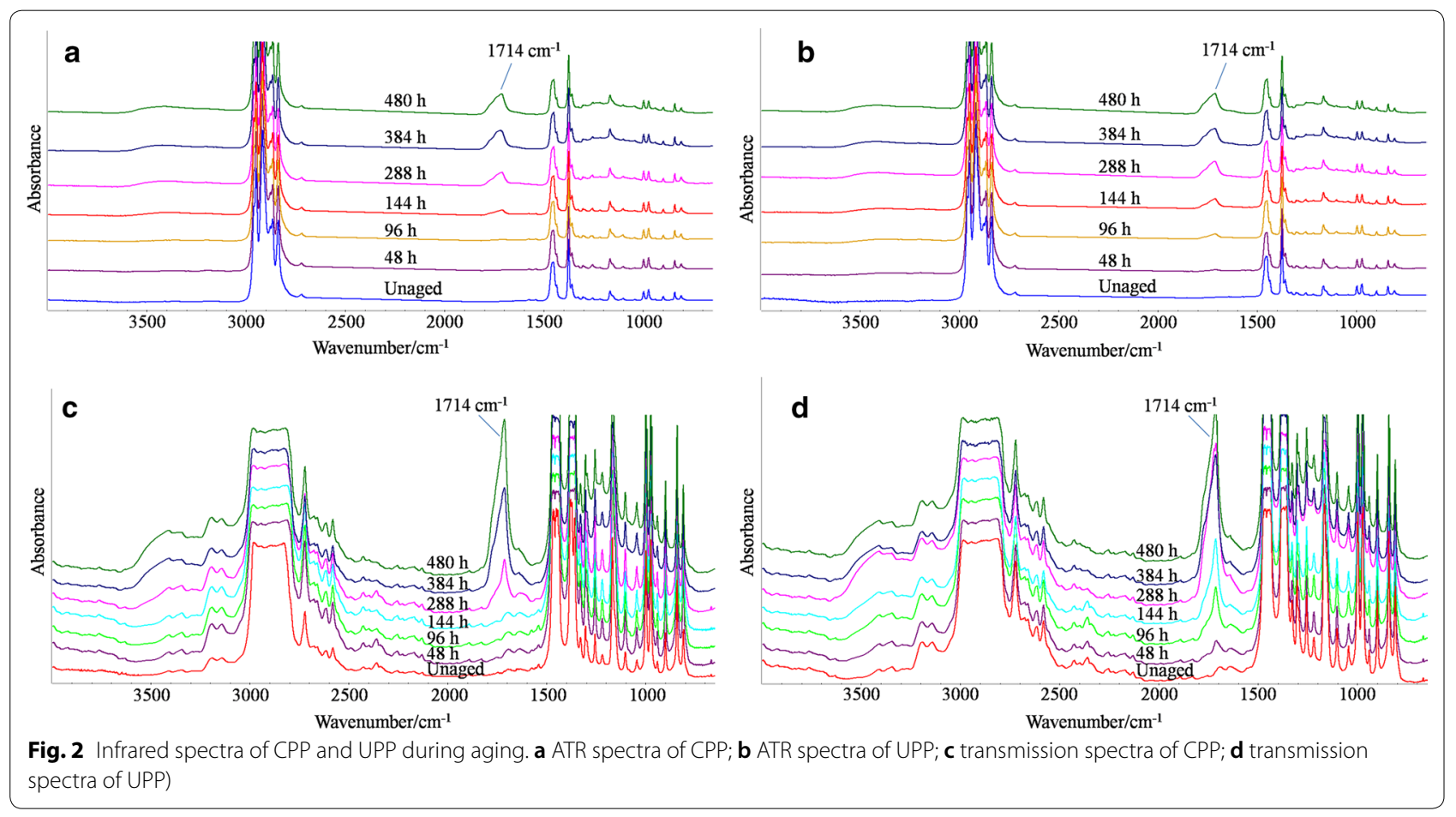



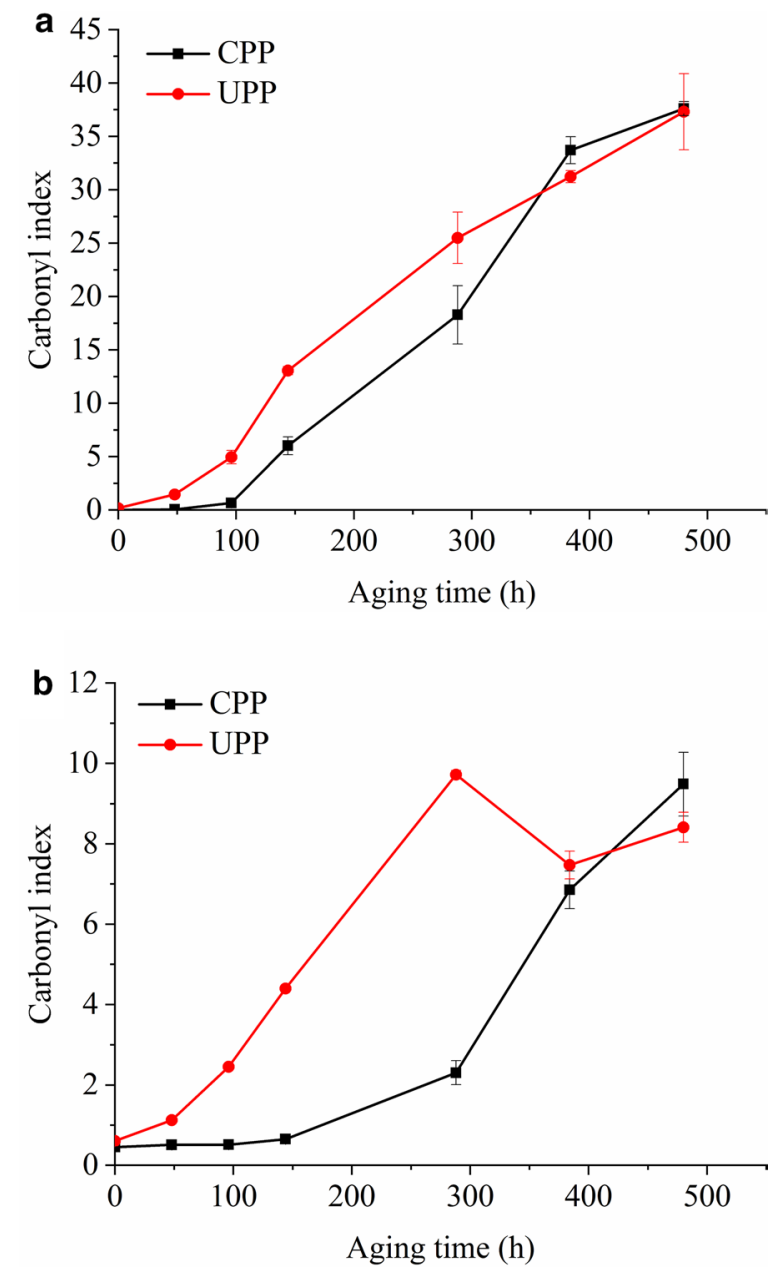

Fig. 3 Carbonyl index of CPP and UPP with aging time. a calculated from ATR spectra; $\mathbf{b}$ calculated from transmission spectra

\section{Volatile degradation products}

Volatile degradation products were absorbed in PP film as liquid. They could be desorbed through flash evaporation and detected by Py-GC/MS. Four typical volatile products, i.e. two alkenes, acetic acid and a lactone, were identified in the flash evaporation-gas chromatograms of two PPs. The peak areas of these products with aging time are shown in Fig. 4. In Fig. 4a, b, the alkenes were generated from the very beginning of aging in UPP, while later than $96 \mathrm{~h}$ in CPP. After a period of time, i.e. $96 \mathrm{~h}$ in UPP and $288 \mathrm{~h}$ in CPP, the peak areas of two alkenes turned to decreasing, corresponding to the increasing of two oxidized products in Fig. $4 \mathrm{c}, \mathrm{d}$ at the same time. This indicated that: (1) The formation of alkenes was prior to the formation of oxidized products, in other words, the chain scission of PP happened before the formation of volatile oxidized products; (2) The oxidized products might be from the further oxidation of alkenes.
The peak areas of volatile oxidized products were expected to keep increasing with aging time due to the continuous oxidation of polymer chains. In CPP, they kept increasing indeed. In UPP, however, the peak areas stopped increasing and even began to decrease from $288 \mathrm{~h}$. This was caused by the evaporation of these products and then condensation on the tube wall. There were visible droplets on the tube wall in which UPP was aged for 384 and $480 \mathrm{~h}$. The droplets were washed by ethanol and the obtained solution was analyzed by Py-GC/MS. The flash evaporation-gas chromatogram of the droplets was compared with the result of the corresponding film (Fig. 5). Acetic acid and the lactone were identified in both the droplets and the corresponding film. Once the volatile oxidized products were evaporated and condensed on the tube wall, they could not come back to the film, so the peak areas in UPP were decreased obviously (Fig. 4c, d).

For CPP, no visible droplets were observed even after aging for $480 \mathrm{~h}$, due to the relative low concentration of volatile oxidized products. There was a maximal concentration of volatile oxidized products that could be retained in the film. If the concentration did not exceed the maximum, the volatile oxidized products would accumulate in the solid polymer. When the concentration exceeded the maximum, the excessive parts began to be desorbed and some condensed on the tube wall. In this case, the biased evaluation of the aging degree of two PPs according to volatile degradation products would also be obtained as according to macromolecular products. Similar phenomenon can be expected if the polymer is photo-oxidized in unsealed atmosphere like in practical use.

\section{Gaseous degradation products}

Gaseous degradation products included $\mathrm{H}_{2}, \mathrm{CO}, \mathrm{CO}_{2}$, alkanes and alkenes. They were in gaseous state and could be identified directly by using GC measurement. The peak areas of four typical gaseous products with aging time are shown in Fig. 6. These products were generated from $48 \mathrm{~h}$ in UPP and from $144 \mathrm{~h}$ in CPP respectively. After that, the peak areas kept increasing in both PPs and the peak areas in UPP were higher than in CPP all along, showing the higher aging degree of UPP without the protection of stabilizers.

\section{Overview of photo-oxidative products in three states}

Induction periods of the generation of photo-oxidative products in three states were extracted from Figs. 3, 4 and 6, listed in Table 1. In CPP, the induction periods of carbonyl products and volatile alkenes were the same, shorter than acetic acid, the lactone and gaseous degradation products, indicating the earlier generation of 

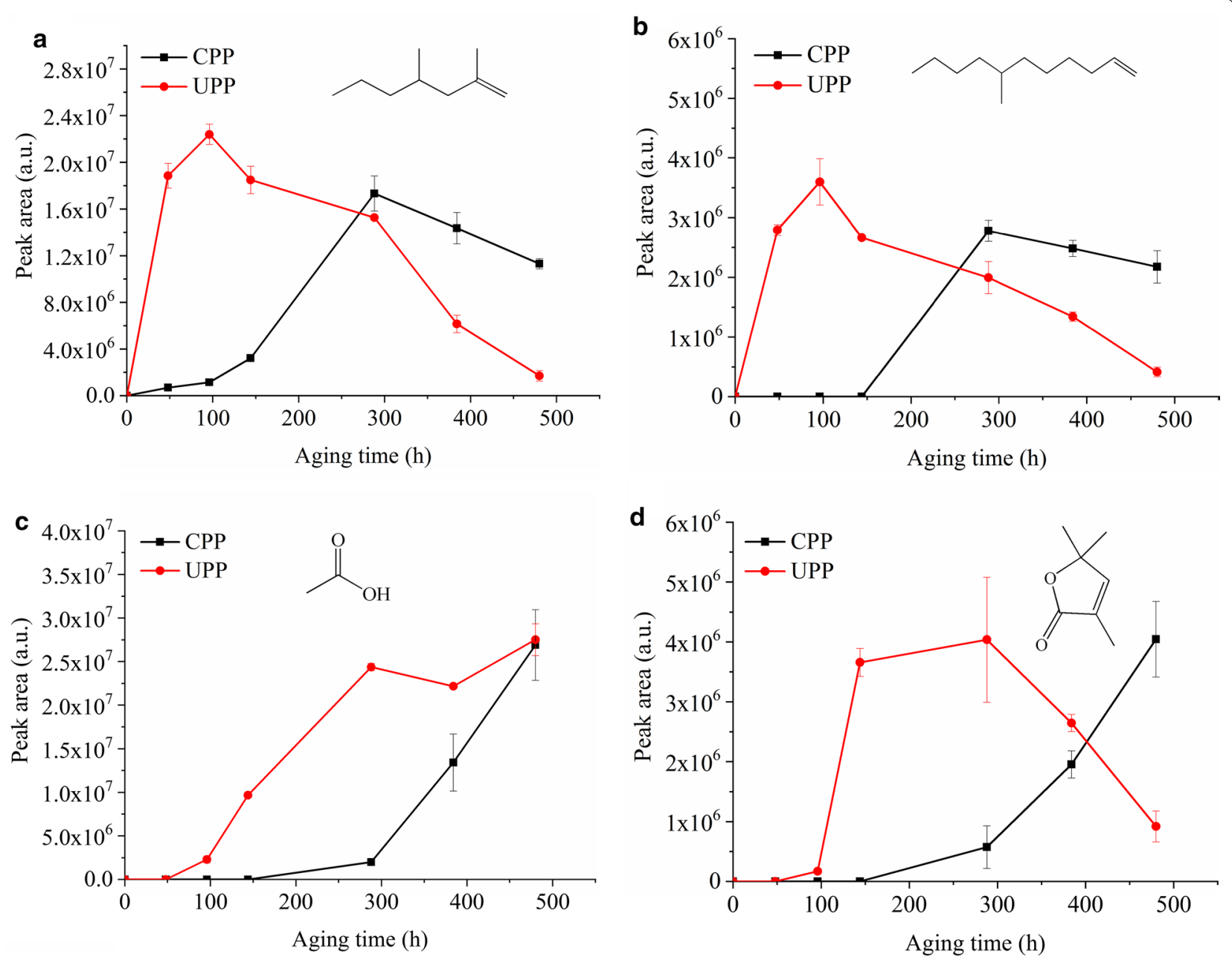

Fig. 4 Peak area of four typical volatile degradation products in CPP and UPP with aging time. $\mathbf{a}$ and $\mathbf{b}$ two alkenes; c acetic acid; $\mathbf{d}$ lactone

oxidation species in macromolecular chains and volatile alkenes. Thereafter volatile alkenes might be further oxidized and the oxidized main chains fractured to generate volatile oxidized products and gaseous degradation products. As shown in Figs. 4c, d and 6, the peak areas of acetic acid, the lactone and gaseous degradation products were increased rapidly from $288 \mathrm{~h}$. At the same time, the peak areas of volatile alkenes were decreased rapidly (Fig. 4a, b). This phenomenon suggested the further oxidation of volatile alkenes and the corresponding accumulation of volatile oxidized products and gaseous degradation products.

Compared with CPP, UPP exhibited much shorter induction periods. Without the protection of stabilizers, oxidation species in macromolecular chains and volatile alkenes were generated from the very beginning of aging, followed by volatile oxidized products and gaseous degradation products within 48 h. As shown in Figs. 3b, 4c, $\mathrm{d}, \mathrm{CI}$ and the peak areas of volatile oxidized products were decreased at the late stage of aging, due to the loss of severely oxidized species in solid state and the migration and condensation of the liquid products. The peak areas of gaseous degradation products kept increasing for as long as $480 \mathrm{~h}$.

Conversion among photo-oxidative products in three states is illustrated in Fig. 7. During the photooxidation of $\mathrm{PP}$, the main chains were first oxidized and the oxidized species remained in the film as solid products. In the meanwhile, the main chains fractured to generate volatile alkenes, absorbed by the film as liquid. Then the oxidized main chains fractured along with the further oxidation of volatile alkenes, to generate volatile oxidized products absorbed by the film as liquid and gaseous degradation products in the atmosphere around the film. The conversion took place throughout the aging process. When the conversion was severe as in UPP, the CI and the concentrations of volatile products were increased first and then 


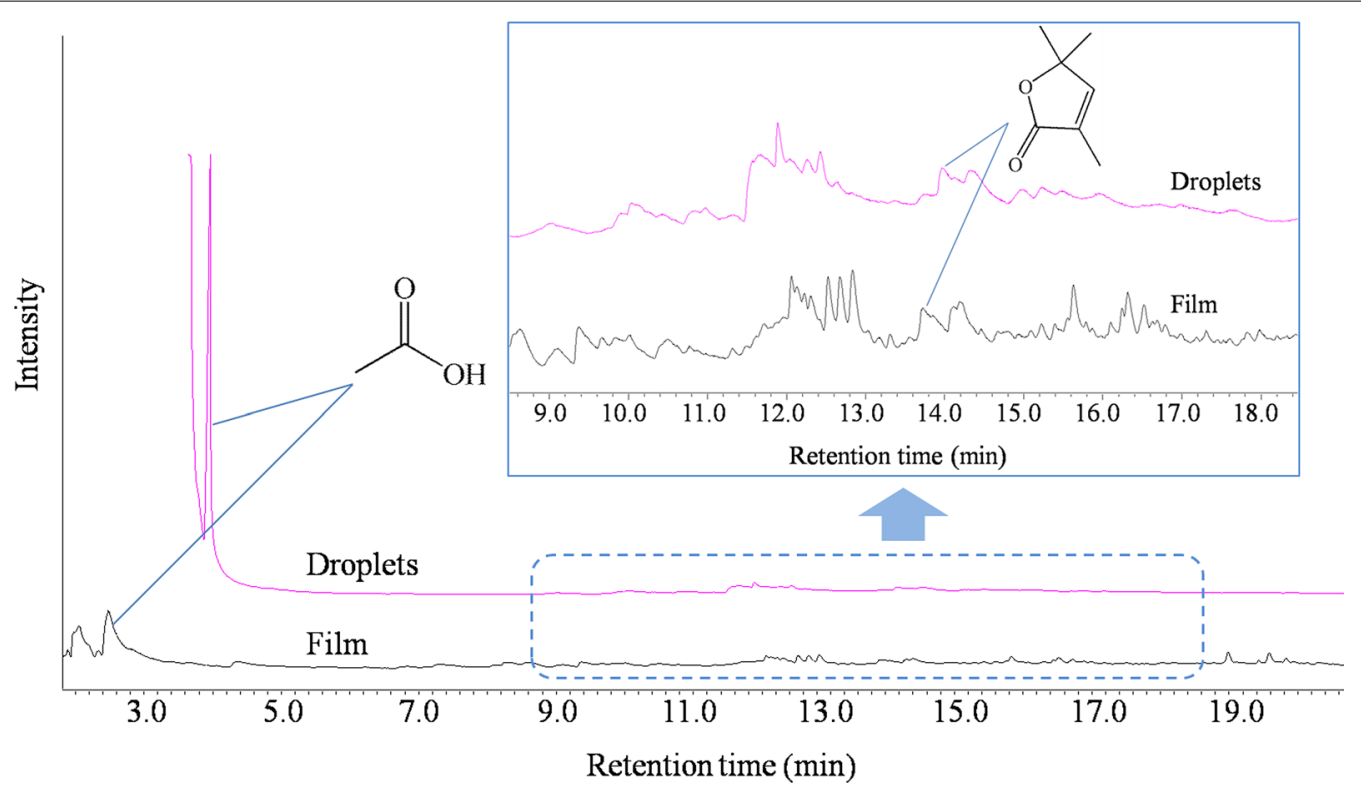

Fig. 5 Flash evaporation-gas chromatograms of UPP and corresponding droplets in the tube after aging for $384 \mathrm{~h}$
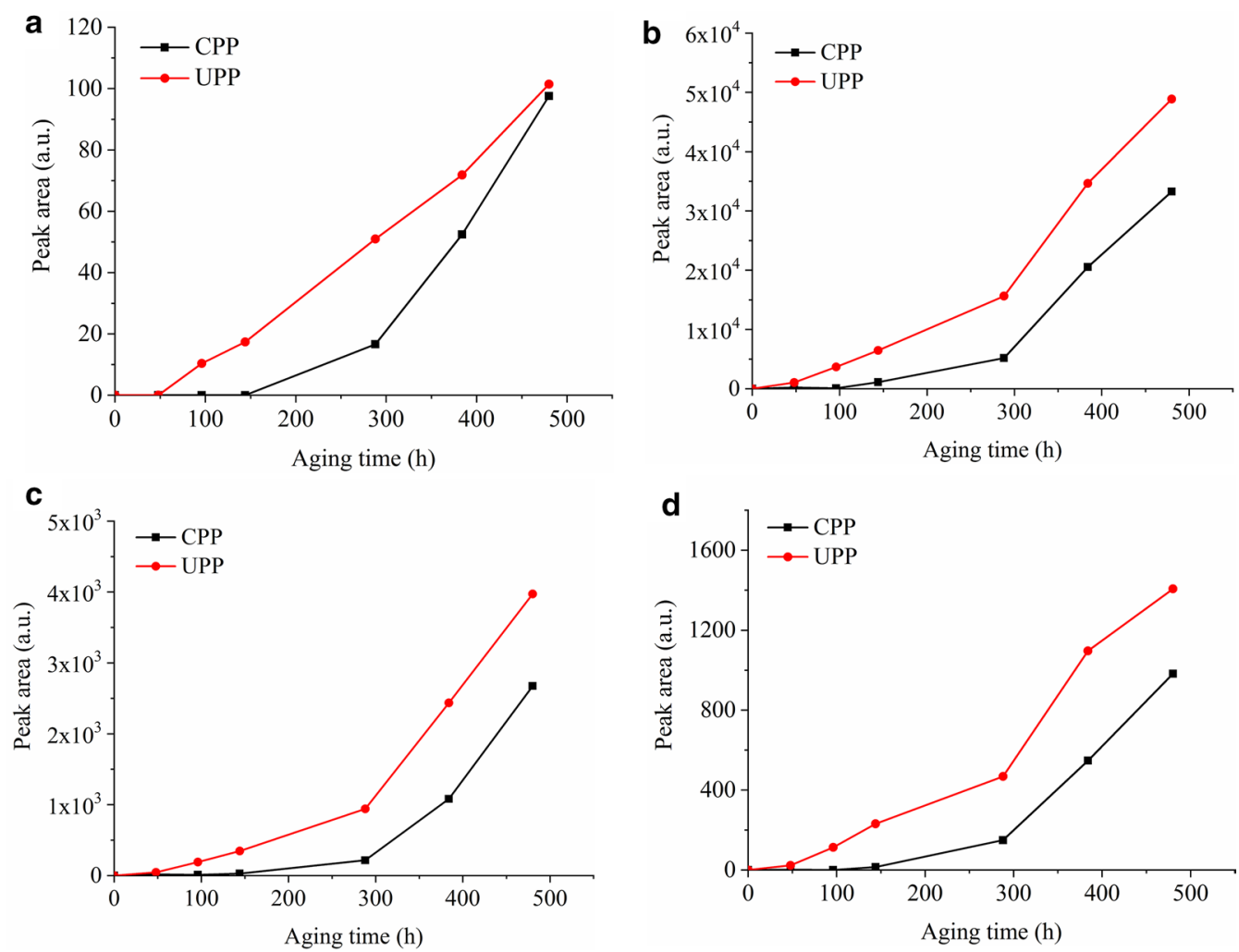

Fig. 6 Peak area of four typical gaseous degradation products in CPP and UPP with aging time. $\mathbf{a} \mathrm{H}_{2} ; \mathbf{b} \mathrm{CO}_{4} \mathbf{c} \mathrm{CH}_{4} ; \mathbf{d} \mathrm{C}_{2} \mathrm{H}_{4}$

decreased, due to the loss of severely oxidized species in solid state and the migration and condensation of liquid droplets in the late stage, despite the continuous aging of the polymer. In CPP, the similar conversion was observed, although the aging process was retarded owing to the presence of stabilizers. Therefore, the 
Table 1 Induction period of generation of photo-oxidative products in CPP and UPP

\begin{tabular}{lcc}
\hline Products & $\begin{array}{l}\text { Induction period } \\
\text { in } \mathbf{C P P / h}\end{array}$ & $\begin{array}{l}\text { Induction } \\
\text { period } \\
\text { in UPP/h }\end{array}$ \\
\hline $\mathrm{Cl}$ on the surface (solid) & 96 & 0 \\
$\mathrm{Cl}$ in the bulk (solid) & 96 & 0 \\
Alkenes (liquid) & 96 & 0 \\
Acetic acid (liquid) & 144 & 48 \\
Lactone (liquid) & 144 & 48 \\
$\mathrm{H}_{2}, \mathrm{CO}, \mathrm{CH}_{4}, \mathrm{C}_{2} \mathrm{H}_{4}$ (gas) & 144 & 48 \\
\hline
\end{tabular}

products in a single state, especially only in solid state, could not reflect the comprehensive aging process and might lead to biased results. It was more reliable to consider the photo-oxidative products in three states as a whole when evaluating the aging degree and relative stability of polymers.

\section{Conclusions}

The photo-oxidative products of CPP and UPP in solid, liquid and gaseous states were detected by FTIR, Py-GC/ MS and GC respectively, and their formation profiles were compared. During photo-oxidative aging, the carbonyl products in macromolecular chains and volatile alkenes were generated first. Then the volatile oxidized products and gaseous degradation products were generated from the further oxidation of volatile alkenes and the fracture of oxidized main chains. At the late stage of aging, $\mathrm{CI}$ and the concentrations of volatile oxidized products were decreased, due to the loss of severely oxidized species in solid state and the migration and condensation of liquid droplets, while the concentrations of gaseous degradation products maintained increasing. Overview of the aging products in three states provides comprehensive information and overall understanding of the aging mechanism. It offers an effective way to evaluate the aging degree and relative stability of polymers accurately.

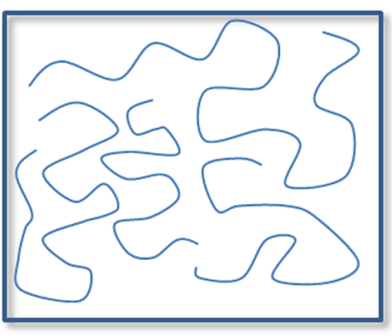

Fracture and oxidation of main chains

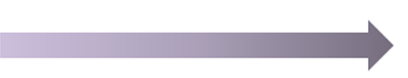

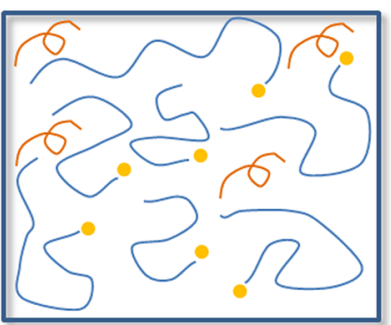

Further oxidation of volatile alkenes and fracture of oxidized main chains
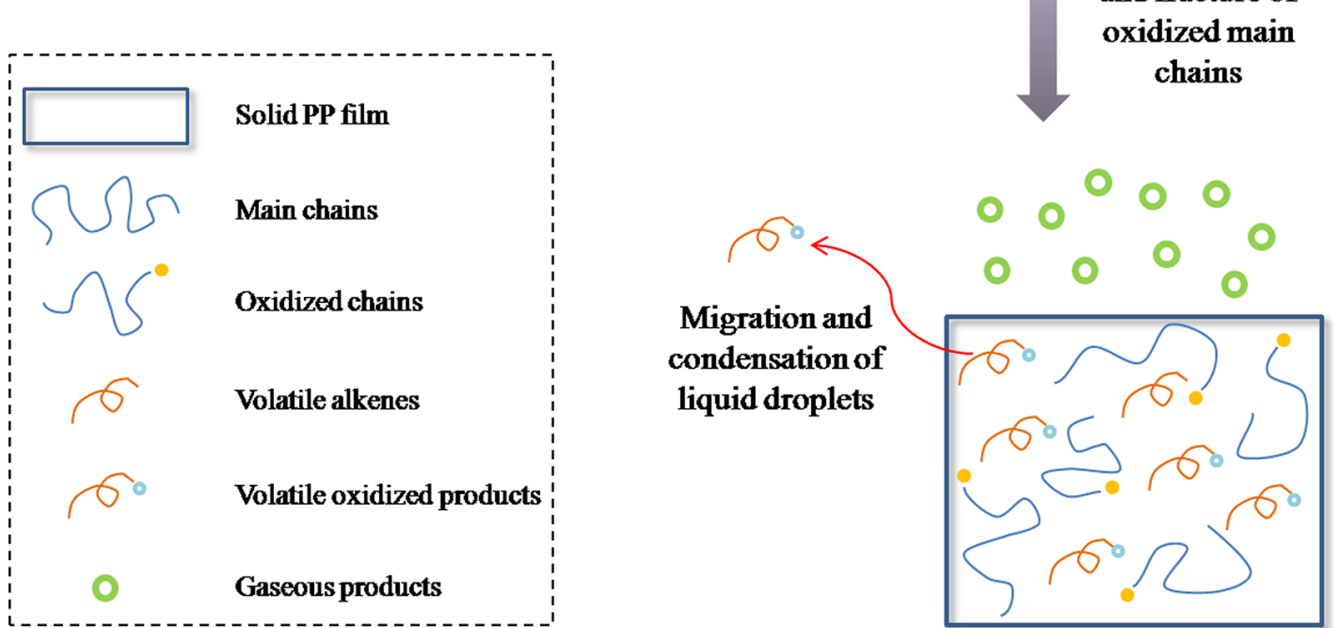

Fig. 7 Schematic diagram of conversion among photo-oxidative products of PP in three states 


\section{Abbreviations}

PP: Polypropylene; CPP: Commercial PP; UPP: Unstabilized PP; FTIR: Fourier transform infrared spectroscopy; ATR: Attenuated total reflection; Py-GC/MS: Pyrolysis-gas chromatography/mass spectrometry; GC: Gas chromatography; $\mathrm{Cl}$ : Carbonyl index.

\section{Acknowledgements}

The authors gratefully acknowledge Jing Li, Chunsong Li and Prof. Qi Lu for their kind help in GC measurements. The authors also thank Guangyan Chen for his kind support in experimental materials.

\section{Authors' contributions}

$\mathrm{XL}$ carried out the experiments and drafted the manuscript. RY proposed the research idea, improved the data interpretation and revised the manuscript. Both authors read and approved the final manuscript.

\section{Funding}

This study was financially supported by the National Natural Science Foundation of China (NSFC, Project No. 51673111). NSFC was not involved in design of the study and collection, analysis, and interpretation of data and in writing the manuscript.

\section{Availability of data and materials}

The datasets used and/or analyzed during the current study are available from the corresponding author on reasonable request.

\section{Competing interests}

The authors declare that they have no competing interests. Rui Yang is currently acting as an Editorial Board Member for BMC Chemistry.

Received: 22 April 2020 Accepted: 15 July 2020

Published online: 18 July 2020

\section{References}

1. Hamid SH (2000) Handbook of polymer degradation. Marcel Dekker, New York, 2nd ed

2. Allen NS, Edge M (1992) Fundamentals of polymer degradation and stabilisation. Elsevier Applied Science, New York

3. Celina MC, Wiggins JS, Billingham NC (eds) (2009) Polymer degradation and performance. American Chemical Society, Washington DC

4. Jelle BP, Nilsen TN (2011) Comparison of accelerated climate ageing methods of polymer building materials by attenuated total reflectance Fourier transform infrared radiation spectroscopy. Constr Build Mater 128:2122-2132

5. Mowery DM, Assink RA, Derzon DK et al (2005) Solid-state ${ }^{13} \mathrm{C}$ NMR investigation of the oxidative degradation of selectively labeled polypropylene by thermal aging and $\gamma$-Irradiation. Macromolecules 38:5035-5046

6. Hakkarainen M, Albertsson A (2005) Indicator products: A new tool for lifetime prediction of polymeric materials. Biomacromol 6:775-779

7. James SL, Robinson AJ, Arnold JC et al (2013) The effects of humidity on photodegradation of poly(vinyl chloride) and polyethylene as measured by the $\mathrm{CO}_{2}$ evolution rate. Polym Degrad Stab 98:508-513

8. Rouillon C, Bussiere PO, Desnoux E et al (2016) Is carbonyl index a quantitative probe to monitor polypropylene photodegradation? Polym Degrad Stab 128:200-208
9. Milichovsky M, Milichovska S (2008) Characterization of oxidized cellulose with ultraviolet-visible spectroscopy. J Appl Polym Sci 107:2045-2052

10. Ahn K, Rosenau T, Potthast A (2013) The influence of alkaline reserve on the aging behavior of book papers. Cellulose 20:1989-2001

11. Komatsu LGH, Oliani WL, Lugao AB et al (2014) Environmental ageing of irradiated polypropylene/montmorillonite nanocomposites obtained in molten state. Radiat Phys Chem 97:233-238

12. Elanmugilan M, Sreekumar PA, Singha $N$ et al (2014) Natural weather aging of low density polyethylene: effect of prodegradant additive. Plast Rubber Compos 43(10):347-353

13. Schwarzinger C, Hintersteiner I, Schwarzinger B et al (2015) Analytical pyrolysis in the determination of the aging of polyethylene. J Anal Appl Pyrol 113:315-322

14. Carlsson DJ, Wiles DM (1969) The photodegradation of polypropylene films II Photolysis of ketonic oxidation products. Macromolecules 2(6):587-597

15. Philippart JL, Posada F, Gardette JL (1995) Mass spectroscopy analysis of volatile photoproducts in photooxidation of polypropylene. Polym Degrad Stab 49:285-290

16. Commereuc S, Vaillant D, Philippart JL et al (1997) Photo and thermal decomposition of iPP hydroperoxides. Polym Degrad Stab 57:175-182

17. Bernstein R, Bernstein SM, Assink RA et al (2007) The origins of volatile oxidation products in the thermal degradation of polypropylene, identified by selective isotopic labeling. Polym Degrad Stab 92:2076-2094

18. Christensen PA, Dilks A, Egerton TA (1999) Infrared spectroscopic evaluation of the photodegradation of paint. J Mater Sci 34:5689-5700

19. Jin CQ, Christensen PA, Egerton TA et al (2006) Rapid measurement of polymer photo-degradation by FTIR spectrometry of evolved carbon dioxide. Polym Degrad Stab 91:1086-1096

20. Jin CQ, Christensen PA, Egerton TA et al (2006) Rapid measurement of photocatalytic oxidation of poly(vinyl chloride) by in situ FTIR spectrometry of evolved $\mathrm{CO}_{2}$. Mater Sci Tech 22(8):908-914

21. Fechine GJM, Christensen PA, Egerton TA et al (2009) Evaluation of poly(ethylene terephthalate) photostabilisation using FTIR spectrometry of evolved carbon dioxide. Polym Degrad Stab 94:234-239

22. Yang R, Yu J, Liu Y et al (2005) Effects of inorganic fillers on the natural photo-oxidation of high-density polyethylene. Polym Degrad Stab 88:333-340

23. Yang R, Christensen PA, Egerton TA et al (2010) Degradation products formed during UV exposure of polyethylene-ZnO nano-composites. Polym Degrad Stab 95:1533-1541

24. Rabello MS, White JR (1997) The role of physical structure and morphology in the photodegradation behaviour of polypropylene. Polym Degrad Stab 56:55-73

25. Yang R, Wang KH, Yu J et al (2006) Depth profiles of high-density polyethylenes filled with different inorganic fillers during natural photo-oxidation by infrared microscopy. Spectrosc Spect Anal 26(5):821-824

26. Li JF, Yang R, Yu J (2008) Study on natural photo-oxidation degradation of polypropylene nanocomposites by infrared microscopy. Spectrosc Spect Anal 28(8):1758-1762

27. Yakimets I, Lai DW, Guigon M (2004) Effect of photo-oxidation cracks on behaviour of thick polypropylene samples. Polym Degrad Stab 86:59-67

\section{Publisher's Note}

Springer Nature remains neutral with regard to jurisdictional claims in published maps and institutional affiliations. 